\title{
Validity of a Smartphone-Based Application for Determining Sprinting Performance
}

\author{
Robert Stanton, Melanie Hayman, Nyree Humphris, Hanna Borgelt, \\ Jordan Fox, Luke Del Vecchio, and Brendan Humphries
}

School of Medical and Applied Sciences, Central Queensland University, Bruce Highway, Rockhampton, QLD 4702, Australia

Correspondence should be addressed to Robert Stanton; r.stanton@cqu.edu.au

Received 9 March 2016; Revised 2 June 2016; Accepted 30 June 2016

Academic Editor: António Ascensão

Copyright (c) 2016 Robert Stanton et al. This is an open access article distributed under the Creative Commons Attribution License, which permits unrestricted use, distribution, and reproduction in any medium, provided the original work is properly cited.

\begin{abstract}
Recent innovations in smartphone technology have led to the development of a number of applications for the valid and reliable measurement of physical performance. Smartphone applications offer a number of advantages over laboratory based testing including cost, portability, and absence of postprocessing. However, smartphone applications for the measurement of running speed have not yet been validated. In the present study, the iOS smartphone application, SpeedClock, was compared to conventional timing lights during flying $10 \mathrm{~m}$ sprints in recreationally active women. Independent samples $t$-test showed no statistically significant difference between SpeedClock and timing lights $(t(190)=1.83, p=0.07)$, while intraclass correlations showed excellent agreement between SpeedClock and timing lights (ICC $(2,1)=0.93, p=0.00,95 \%$ CI $0.64-0.97$ ). Bland-Altman plots showed a small systematic bias (mean difference $=0.13$ seconds) with SpeedClock giving slightly lower values compared to the timing lights. Our findings suggest SpeedClock for iOS devices is a low-cost, valid tool for the assessment of mean flying $10 \mathrm{~m}$ sprint velocity in recreationally active females. Systematic bias should be considered when interpreting the results from SpeedClock.
\end{abstract}

\section{Introduction}

The use of emerging technologies such as smartphones and tablets offers researchers and coaches opportunities to undertake physical performance assessments in the field, rather than the sports science laboratory. Current smartphone technology includes advanced computing capacity, inertial sensors, a global positioning system, and high speed video capacity [1]. Recently developed smartphone applications (APPs) for sport have capitalised on these advances and are shown to be valid and reliable tools to assess lower limb functional performance during countermovement jump [2, $3]$, drop jump [3], balance $[4,5]$, and maximal strength [6] tests. However, while the assessments of jumping performance and muscular strength are important characteristics of human performance, sprint speed is critical for success in a range of activities including football [7] and netball [8]. Yet unlike other physical performance characteristics, the assessment of sprinting capacity has received relatively less attention in the peer-reviewed literature [9].
Although fully automated timing systems using pressure sensitive starting blocks or photo finish recording systems remain the gold standard for the assessment of sprint running performance, such systems are rarely found outside athletic venues. They lack portability and are cost prohibitive to many teams, coaches, and athletes. Dual beam photocell systems are increasingly used due to their lower cost, portability, and capability to wirelessly send data to a handheld receiver. Dual beam systems exhibit greater accuracy compared to singlebeam systems and have been recommended over singlebeam systems [10]. Recent developments in error detection algorithms to address some of the shortcomings associated with single-beam systems have shown promising results. For example, the SmartSpeed (Fusion Sport, Coopers Plains, Australia), single-beam system with error detection, has been shown to comply with Australia's National Sport Science Quality Assurance standards, which require sprint testing systems to achieve a maximal typical error of 0.05 seconds over $30 \mathrm{~m}$. D'Auria and colleagues [11] showed that the SmartSpeed system achieved a typical error of $\leq 0.03$ seconds at distances of 5,10 , and $20 \mathrm{~m}$. 
Studies also show that video analysis of running performance has been shown to closely match fully automated timing systems [12] and dual beam photocell systems [13], even when recorded at 50 or $100 \mathrm{~Hz}$. Therefore, even low-speed video capture may represent a low-cost and portable option to assess running performance. Recent innovations in smartphone technology have seen video capture speeds up to $240 \mathrm{~Hz}$ on iPhone 6. Coupled with improved image detection algorithms and computing power, smartphone APPs have been developed to allow coaches and researchers to determine running speed using these popular and low-cost tools. One such APP is SpeedClock (http://appmaker.se/?m=5\&s=0). SpeedClock uses the iOS device camera to detect and record motion and, with the user input of a reference distance, calculates speed. SpeedClock can be used on a single standalone device, with the reference frame defined by the edges of the image detection field, or connected via Bluetooth with a second iOS device to record time over longer distances.

Although APPs such as SpeedClock are purported to accurately record sprint performance, to the best of our knowledge, there are currently no studies which demonstrate the validity of SpeedClock. Such studies are important in confirming the usefulness of emerging technologies and are of great interest to athletes, coaches, and researchers. Therefore, the purpose of the present study is to examine the validity of the SpeedClock APP using timing lights as a reference.

\section{Methods}

2.1. Participants. Participants were a convenience sample of 24 recreationally active females ( $>18$ years) recruited via personal discussion with investigators. An information sheet outlining the purpose, risks, and benefits of participation was provided to all potential participants. Prior to inclusion in the study, participants were screened for musculoskeletal, neurological, or cardiorespiratory concerns which would contraindicate the performance of maximal sprinting, using stage one of the Adult Preexercise Screening System [14]. Written informed consent was obtained from all participants prior to the commencement of data collection. This study was approved by the institutional human research ethics committee.

2.2. Protocols. Participants stature was measured using a Seca 213 portable stadiometer (Seca GMBH, Hamburg) and weight determined using a Seca robusta 813 portable scale (Seca GMBH, Hamburg). All measures were performed according to standardised protocols [15]. All participants underwent a standardised warm-up comprising five minutes of light jogging, static and dynamic stretching, and a series of submaximal sprints. Participants then performed four maximal effort $20 \mathrm{~m}$ sprints, separated by five minutes of passive rest. Participants started on a line between the first set of timing lights and commenced the sprint in their own time. Standardised instructions were provided regarding maximal effort and to decelerate only when past the final set of timing

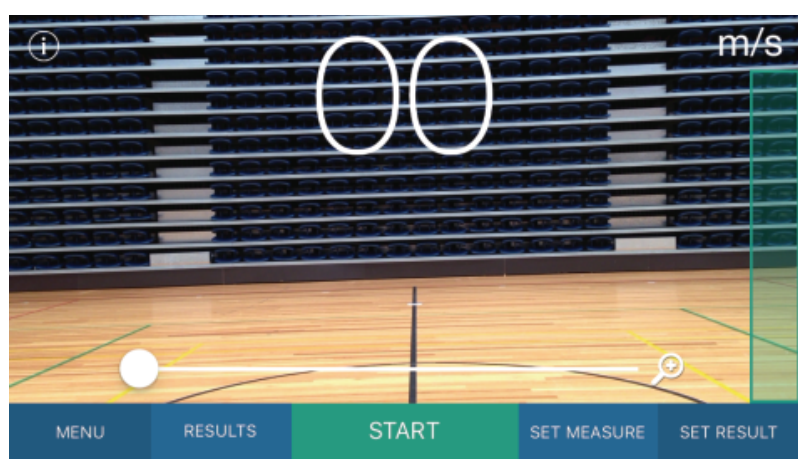

FIGURE 1: Screenshot of iPhone $5 c$ showing motion detection zones at each edge of the field of view.

lights. All sprints were performed in an indoor sports stadium $\left(\sim 26^{\circ} \mathrm{C}, 50 \% \mathrm{RH}\right)$ on a suspended timber floor. Participants wore their usual running shoes and apparel.

2.3. Instruments. Sprint times were recorded using SmartSpeed Pro timing lights (Fusion Sport, Coopers Plains, Australia), with gates at zero, 10, and 20 metres. This system uses a single-beam design to improve battery life and ease of setup, however, incorporates novel error detection algorithms to reduce false triggers. In the event of multiple triggers, the algorithm interprets the longest trigger as the true start time. Gates were set at a height of $1.0 \mathrm{~m}$ from the floor. Sprint times were converted to mean sprint velocity $(\mathrm{m} / \mathrm{s})$ for $0-10 \mathrm{~m}, 10$ $20 \mathrm{~m}$, and $0-20 \mathrm{~m}$ using a standard linear motion equation $(v=d / t)$. To examine the validity of the SpeedClock iOS application, the APP was installed on an iPhone $5 c$ running iOS Version 9.2.1 (Apple Corporation, Cupertino, CA), which records video at 60 frames per second. In SpeedM (Motion) mode, the APP incorporates motion detection zones at each edge of the image which trigger and terminate data collection (Figure 1). For the present study, to ensure repeatability of the method and to minimise movement of the device, iPhone was mounted in a plastic tripod mount on a Velbon EX 330 tripod (Velbon Corporation, Tokyo, Japan), with the camera lens $1.0 \mathrm{~m}$ from the ground. The tripod was positioned $10.5 \mathrm{~m}$ perpendicular to the midpoint between the timing lights at 10 and $20 \mathrm{~m}$, such that the motion detection zones of the application were aligned with the 10 and $20 \mathrm{~m}$ timing lights. The tracking sensitivity on the APP was set to 0.92 (arbitrary units) to avoid false triggers from background movement. In this manner, as the participant ran through the timing lights at $10 \mathrm{~m}$, motion detection triggered data acquisition. Similarly, as the participant ran through the timing lights at $20 \mathrm{~m}$, motion detection terminated data acquisition. Figure 2 shows the positioning of the timing lights and iOS device. Mean velocity $(\mathrm{m} / \mathrm{s})$ for the flying $10 \mathrm{~m}$ sprint was displayed on-screen and manually recorded for later analysis.

2.4. Statistical Analysis. Descriptive statistics were calculated for participant data. All four flying $10 \mathrm{~m}$ sprints recorded simultaneously using timing lights and the SpeedClock application were used for analysis. Independent sample $t$-tests 


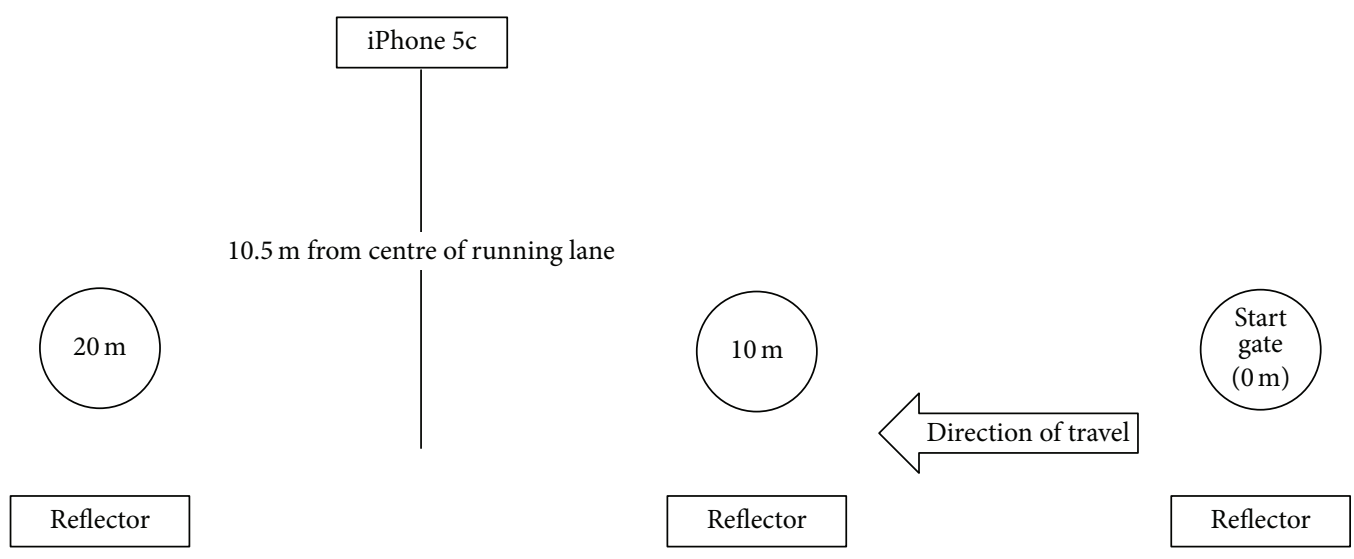

FIgure 2: Positioning of the timing lights and iOS device.

TABLE 1: Mean flying $10 \mathrm{~m}$ sprint times for timing lights and SpeedClock APP.

\begin{tabular}{lc}
\hline Timing lights & SpeedClock APP \\
\hline $6.48 \pm 0.49$ & $6.31 \pm 0.48$ \\
\hline
\end{tabular}

(two-tailed) were used to identify differences in average velocity obtained from the timing lights and SpeedClock APP. Between-device agreement was examined using intraclass correlation coefficients (ICC 2,1) with 95\% confidence intervals (95\% CI) and interpreted according to Munro [16]. Bland-Altman plots were then constructed using Microsoft Excel (Microsoft Corporation, Redmond, USA) to visualise the level of agreement between average velocity obtained from the timing lights and the SpeedClock APP. With the exception of Bland-Altman plots, all statistical analyses were performed using Statistical Package for the Social Sciences (SPSS), Version 22 (IBM Corporation, Chicago, Ill). Statistical significance was accepted at an alpha level of $p<0.05$.

\section{Results}

Twenty-four recreationally active females (mean age $26.6 \pm$ 5.4 years, mean body mass index $25.0 \pm 3.1 \mathrm{~kg} \cdot \mathrm{m}^{-2}$ ) provided informed consent and participated in the study. No adverse events were reported. Results for mean flying $10 \mathrm{~m}$ sprint times are shown in Table 1 . Independent samples $t$ test showed no statistically significant difference in mean flying $10 \mathrm{~m}$ sprint velocity between data from the timing lights $(6.47 \pm 0.49 \mathrm{sec})$ and the SpeedClock APP (6.31 \pm $0.48 \mathrm{sec})(t(190)=1.83, p=0.07)$. Intraclass correlations (ICC 2,1) showed excellent agreement in mean flying $10 \mathrm{~m}$ sprint velocity between data from the timing lights and the SpeedClock APP $(\operatorname{ICC}(2,1)=0.93, p=0.00,95 \%$ CI $0.64-0.97)$. Bland-Altman plots to visualise the difference between mean flying $10 \mathrm{~m}$ sprint velocity determined by timing lights and SpeedClock APP are shown in Figure 3. A small systematic bias (mean difference $=0.13$ seconds) shows the APP consistently gave slightly lower values compared to the timing lights.

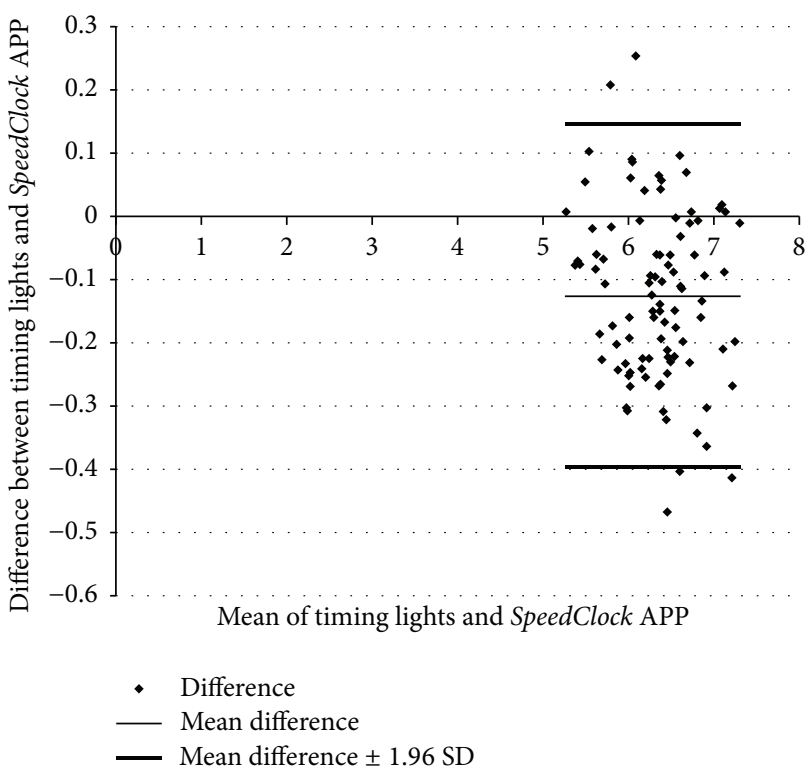

Figure 3: Bland-Altman plot depicting the level of agreement between timing lights and SpeedClock application for $10 \mathrm{~m}$ sprinting.

\section{Discussion}

To the best of our knowledge, no other studies comparing APP-based measures of sprint running speed have been published in the peer-reviewed literature. As such this study makes an important contribution to the exercise and sports science domain. The findings from the present study suggest that the SpeedClock APP installed on an iPhone $5 \mathrm{c}$ recording video at 60 frames per second is a valid measure of average flying $10 \mathrm{~m}$ sprint velocity in recreationally active females.

A number of other studies have examined the use of video technology in determining sprint running performance. For example, Haugen and colleagues [12] reported no systematic variation between Brower dual beam infrared timing and Dartfish-based video analysis of $40 \mathrm{~m}$ sprint times in national level male and female track athletes. In another study, Harrison and colleagues [13] reported the validity of video recorded at $50 \mathrm{~Hz}$ and at $100 \mathrm{~Hz}$, to determine 
mean sprint velocity over $3 \mathrm{~m}$. No statistically significant differences and excellent ICCs were observed between a laser sports measurement system and video recorded at either frame rate. However, at medium to fast sprinting velocities, video recorded at $100 \mathrm{~Hz}$ compared more favorably with laser-derived measures than video recorded at $50 \mathrm{~Hz}$, due to the higher sampling rate. In the present study, mean flying $10 \mathrm{~m}$ sprit velocity recorded using the SpeedClock APP was not significantly different to that recorded using dual beam timing lights. Moreover, ICCs showed excellent agreement with Bland-Altman plots indicating only a small degree of systematic bias, with a mean difference of -0.13 seconds (1.97\%). Although this level of mean difference may be significant with respect to changes in sprint performance over time, these values are likely within expected test-retest reliability of sprinting performance, and researchers should examine the smallest worthwhile change in performance to determine if a real change has occurred.

One notable difference between the timing lights and the SpeedClock APP is that the timing lights report data to three decimal places, while the SpeedClock APP reports data to two decimal places. Therefore, we reran our ICC analysis using velocities based on timing light data under similar conditions, firstly truncated and then rounded to two decimal places. The resultant analyses were not substantially different from our initial findings (ICC $(2,1)=0.92, p=0.00,95 \%$ CI $0.68-$ 0.97 , and $0.93, p=0.00,95 \%$ CI $0.64-0.97$, resp.) and do not change our interpretation of the validity of this APP.

Emerging technologies such as the use of smartphone applications in sport have the potential to provide athletes, coaches, and researchers with additional data not otherwise available with a single laboratory based system. For example, when using SpeedClock, an image of the participant can be captured midway through the image capture field. This may provide valuable data to assess running technique, and, since the image is stamped with the mean velocity, it serves as a permanent record of the attempt. Unlike GPS-based system, SpeedClock is video-based and therefore can be used indoors. Finally, unlike manual stopwatch timing, the data is not affected by parallax error when standing at the start or finish line, and in the case of SpeedClock, two devices can be used together in a manner not unlike timing gates. In general, smartphone-based APPs for sprint performance assessment offer low-cost, portability, and ease of use, not otherwise available with laboratory based systems, and their widespread applicability to training, rehabilitation, and research warrants further investigation.

A strength of the present study is the use of Bland-Altman plots to examine systematic bias between methods used to determine sprint velocity. However, a potential limitation is the use of the iPhone $5 \mathrm{c}$, which records video at 60 frames per second. Future studies should examine the use of high speed video on newer iOS devices as the increased resolution afforded by the higher frame rate may improve accuracy and reduce systematic bias. Future studies should also examine other speed recording APPs on Android operating systems, since, at the present time, SpeedClock is only available on iOS and, although iOS devices are widely utilised, they are not the sole software platform. Finally, future studies should examine the validity of the SpeedClock or similar APPs over shorter and longer distances and in clinical populations.

\section{Conclusions}

SpeedClock for iOS devices is a low-cost, valid tool for the assessment of mean flying $10 \mathrm{~m}$ sprint velocity in recreationally active females. Further studies are required in different populations and settings to generalise the findings from the present study.

\section{Competing Interests}

There is no conflict of interests to declare.

\section{References}

[1] M. B. del Rosario, S. J. Redmond, and N. H. Lovell, “Tracking the evolution of smartphone sensing for monitoring human movement," Sensors, vol. 15, no. 8, pp. 18901-18933, 2015.

[2] C. Balsalobre-Fernández, M. Glaister, and R. A. Lockey, “The validity and reliability of an iPhone app for measuring vertical jump performance," Journal of Sports Sciences, vol. 33, no. 15, pp. 1574-1579, 2015.

[3] F. Gallardo-Fuentes, J. Gallardo-Fuentes, R. Ramírez-Campillo et al., "Inter and intra-session reliability and validity of the My Jump app for measuring different jump actions in trained male and female athletes," The Journal of Strength and Conditioning Research, vol. 30, no. 7, pp. 2049-2056, 2016.

[4] R. Z. Amick, A. Chaparro, and J. A. Patterson, "Test-retest reliability of the sway balance mobile application," Journal of Mobile Technology in Medicine, vol. 4, no. 2, pp. 40-47, 2015.

[5] J. A. Patterson, R. Z. Amick, T. Thummar et al., "Validation of measures from the smartphone SWAY balance application: a pilot study," International Journal of Sports Physical Therapy, vol. 9, no. 2, pp. 135-139, 2014.

[6] C. Balsalobre-Fernández, M. Kuzdub, P. Poveda-Ortiz, and J. D. Campo-Vecino, "Validity and reliability of the push wearable device to measure movement velocity during the back squat exercise," The Journal of Strength \& Conditioning Research, vol. 30, no. 7, pp. 1968-1974, 2016.

[7] O. Faude, T. Koch, and T. Meyer, "Straight sprinting is the most frequent action in goal situations in professional football," Journal of Sports Sciences, vol. 30, no. 7, pp. 625-631, 2012.

[8] J. K. Hewit, J. B. Cronin, and P. A. Hume, "Kinematic factors affecting fast and slow straight and change-of-direction acceleration times," The Journal of Strength \& Conditioning Research, vol. 27, no. 1, pp. 69-75, 2013.

[9] T. Haugen and M. Buchheit, "Sprint running performance monitoring: methodological and practical considerations," Sports Medicine, vol. 46, no. 5, pp. 641-656, 2016.

[10] T. A. Haugen, E. Tønnessen, I. S. Svendsen, and S. Seiler, "Sprint time differences between single- and dual-beam timing systems," The Journal of Strength \& Conditioning Research, vol. 28, no. 8, pp. 2376-2379, 2014.

[11] S. D’Auria, R. Tanner, J. Sheppard et al., "Evaluation of various methodologies used to assess sprint performance," in Proceedings of the Australian Institute of Sport Applied Physiology Conference, ACT, Canberra, Australia, 2006. 
[12] T. A. Haugen, E. Tønnessen, and S. K. Seiler, "The difference is in the start: Impact of timing and start procedure on sprint running performance," The Journal of Strength and Conditioning Research, vol. 26, no. 2, pp. 473-479, 2012.

[13] A. J. Harrison, R. L. Jensen, and O. Donoghue, "A comparison of laser and video techniques for determining displacement and velocity during running," Measurement in Physical Education and Exercise Science, vol. 9, no. 4, pp. 219-231, 2005.

[14] ESSA, Adult Pre-Exercise Screening System, Exercise and Sports Science, Brisbane, Australia, 2011.

[15] K. Norton and T. Olds, Anthropometrica, UNSW Press, Sydney, Australia, 1996.

[16] B. Munro, Statistical Methods for Health Care Research, J. B. Lippincott, Philadelphia, Pa, USA, 1997. 


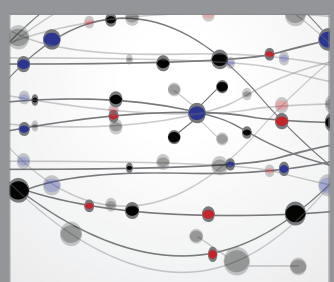

The Scientific World Journal
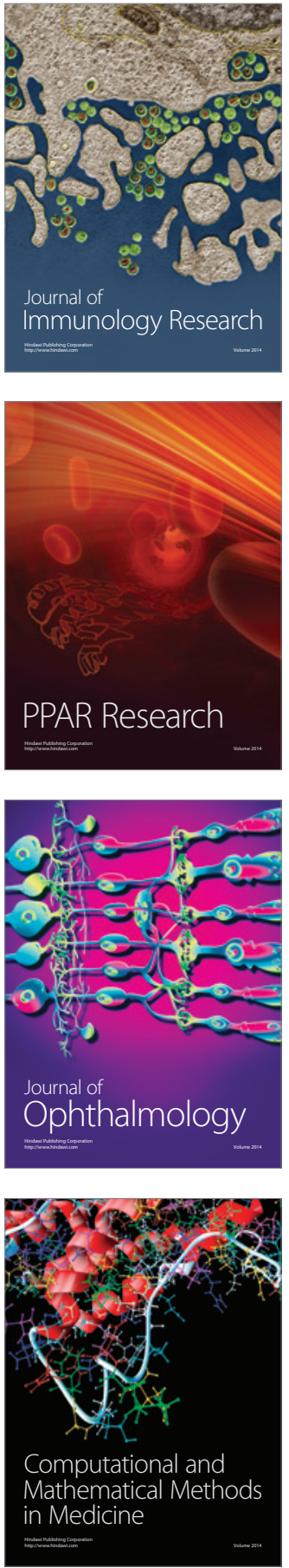

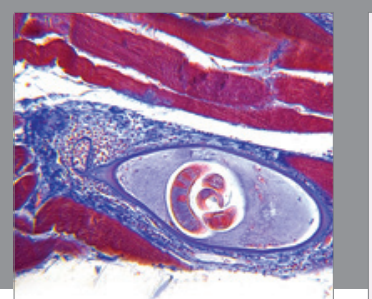

Gastroenterology Research and Practice

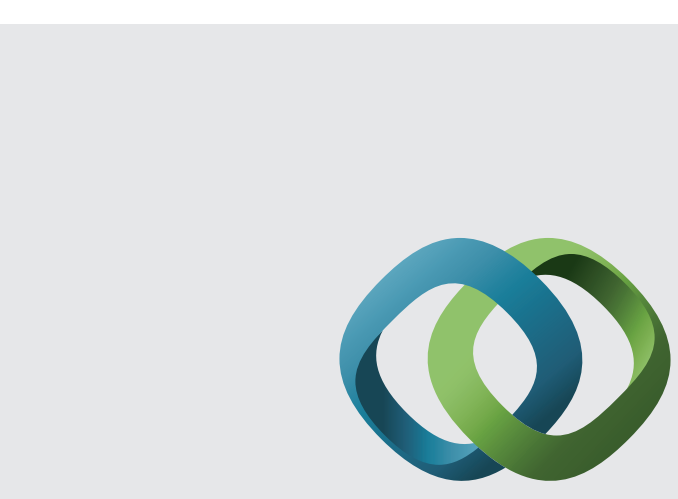

\section{Hindawi}

Submit your manuscripts at

http://www.hindawi.com
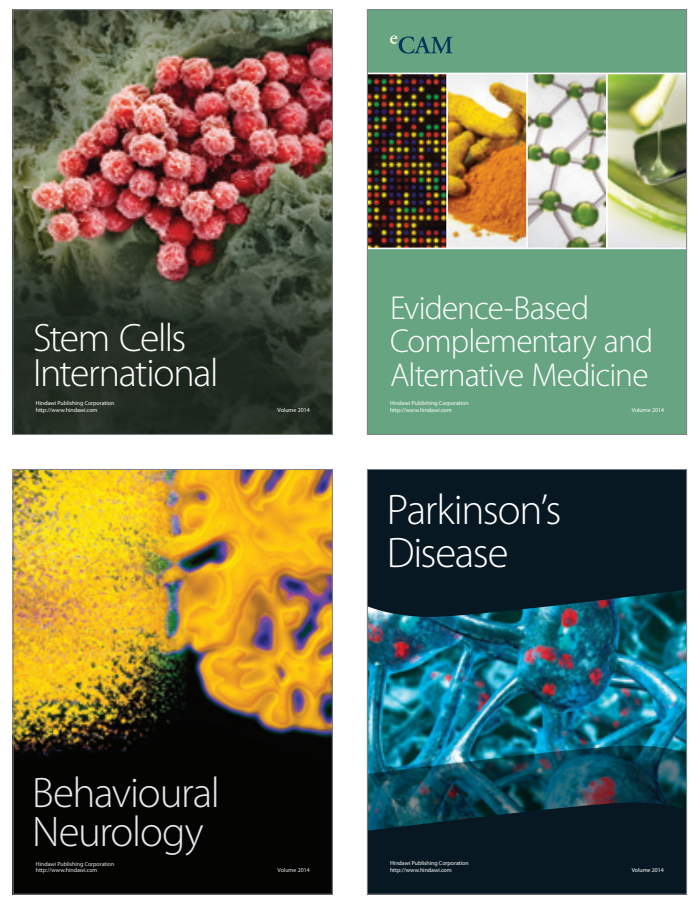
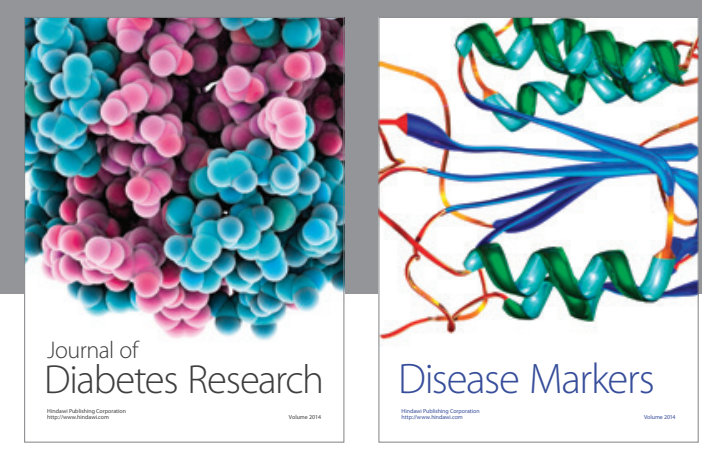

Disease Markers
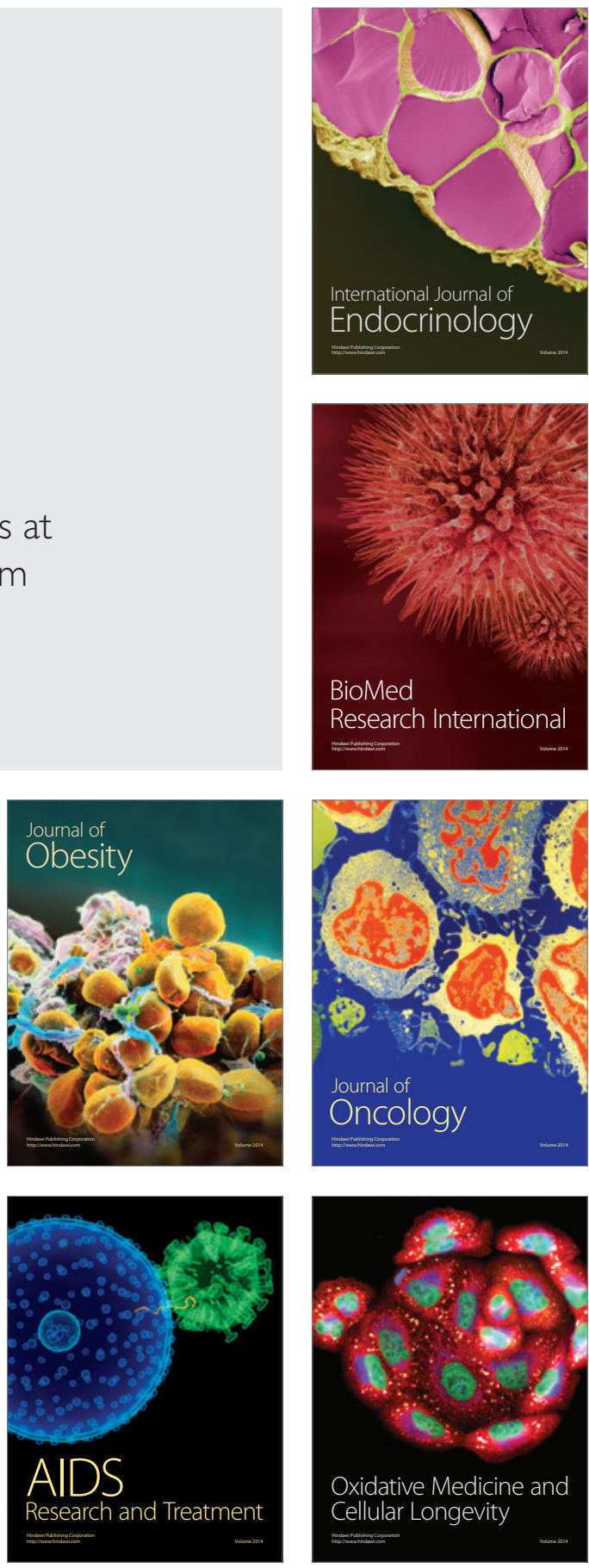